\title{
Aggrecan Glycoforms Contribute to the Molecular Heterogeneity of Perineuronal Nets
}

\author{
Russell T. Matthews, ${ }^{1}$ Gail M. Kelly, ${ }^{1}$ Cynthia A. Zerillo, ${ }^{1}$ Grace Gray, ${ }^{1}$ Michael Tiemeyer, ${ }^{2}$ and \\ Susan Hockfield ${ }^{1}$ \\ ${ }^{1}$ Department of Neurobiology, Yale University School of Medicine, New Haven, Connecticut 06520, and ²Glyko, Inc., \\ Novato, California, 94949
}

The perineuronal net forms the extracellular matrix of many neurons in the CNS, surrounding neuron cell bodies and proximal dendrites in a mesh-like structure with open "holes" at the sites of synaptic contacts. The perineuronal net is first detected late in development, approximately coincident with the transformation of the CNS from an environment conducive to neuronal growth and motility to one that is restrictive, suggesting a role for the perineuronal net in this developmental transition. Perineuronal nets show a great degree of molecular heterogeneity. Using monoclonal antibodies Cat-301, Cat-315, and Cat316, we have shown previously that although all antibodies recognize chondroitin sulfate proteoglycans of similar sizes, each antibody recognizes perineuronal nets on distinct but overlapping sets of neurons in the adult cat CNS. An understanding of the heterogeneity demonstrated by these antibodies is critical to understanding the organization and function of

The perineuronal net forms the extracellular matrix (ECM) of many neurons in the CNS. It surrounds neuronal cell bodies and proximal dendrites in a mesh-like structure with open "holes" at the sites of synaptic contacts (Hockfield and McKay, 1983; Zaremba et al., 1989; Celio and Blumcke, 1994; Celio et al., 1998). Perineuronal nets have been visualized using a variety of techniques, including immunohistochemical stains that label polyanionic molecules such as glycosaminoglycan (GAG) chains (Rambourg et al., 1966; Bondareff, 1967; Castejon, 1970), lectins that bind $N$-acetylgalactosamine (Nakagawa et al., 1986; Bruckner et al., 1993; Schweizer et al., 1993), and monoclonal antibodies to chondroitin sulfate proteoglycans (CSPGs) (Hockfield and McKay, 1983; Fujita et al., 1989; Watanabe et al., 1989; Bertolotto et al., 1990; Guimaraes et al., 1990; Asher et al., 1995; Wintergerst et al., 1996). The perineuronal net is first detected relatively late in development, as the mature synaptic circuitry of the CNS is established and stabilized. A full understanding of the composition and function of the perineuronal net has remained elusive; however, a role in target recognition and selective synapse formation and stabilization is suggested by the timing of its deposition, its perisynaptic localization, and its molecular heterogeneity.

One extensively characterized component of the perineuronal

Received Feb. 25, 2002; revised May 21, 2002; accepted May 21, 2002.

This work was supported by National Institutes of Health Grant EY06511. We thank Dr. H. Watanabe (National Institute of Dental and Craniofacial Research) for supplying tissue from the $c m d$ mice.

Correspondence should be addressed to Russell T. Matthews, Department of Neurobiology, Yale University School of Medicine, P.O. Box 208001, New Haven, CT 06520. E-mail: russell.matthews@yale.edu.

Copyright (ㄷ) 2002 Society for Neuroscience $0270-6474 / 02 / 227536-12 \$ 15.00 / 0$ perineuronal nets. Using aggrecan knock-out mice ( $\mathrm{cmd}$ ), we have now determined that all three antibodies recognize aggrecan. Chemical and enzymatic deglycosylation show that the differences revealed by the three antibodies arise from differential glycosylation of aggrecan. We further demonstrate that aggrecan mRNA is expressed relatively late in development and that neurons themselves are likely the predominant cellular sites of aggrecan expression. This work indicates that neurons can directly regulate the composition of their extracellular matrix by regulated synthesis and differential glycosylation of aggrecan in a cell type-specific manner. These results have important implications for the role of regulated microheterogeneity of glycosylation in the CNS.

Key words: chondroitin sulfates; proteoglycans; glycosylation; lectican; extracellular matrix; synapse formation

net is the large CSPG recognized by the monoclonal antibody Cat-301 (Zaremba et al., 1989). The Cat-301 CSPG marks the surface of cell bodies and proximal dendrites of specific subsets of neurons in several areas of the mammalian CNS, including the visual cortex and dorsal lateral geniculate nucleus of cats and primates (Hockfield and McKay, 1983; Hockfield et al., 1990). Cat-301 immunoreactivity is first detected relatively late in postnatal development, coinciding with the establishment of mature synaptic connections (Guimaraes et al., 1990; Hockfield et al., 1990). The acquisition of normal levels of Cat-301 expression requires normal patterns of neuronal activity during the early postnatal period and parallels the requirement for normal patterns of activity for the acquisition of mature anatomical and physiological properties of neurons (Kalb and Hockfield, 1988, 1990a,b, 1994; Sur et al., 1988; Guimaraes et al., 1990; Hockfield et al., 1990).

Cat-315 and Cat-316, two more recently characterized monoclonal antibodies, also recognize perineuronal nets on neuronal subsets that overlap with, but are not identical to, the subsets identified by Cat-301 (Lander et al., 1997, 1998). All three antibodies recognize CSPGs of approximately the same apparent molecular weight (Lander et al., 1997). Serial immunodepletion analysis demonstrated that the three Cat-3xx antibodies recognize overlapping but nonidentical CSPGs (Lander et al., 1997), some reactive with all three antibodies, others reactive with any pair of the antibodies, and others reactive with only one of the antibodies. In total, these biochemical analyses provided evidence for at least seven different CSPGs, reactive with combinations of the Cat-3xx antibodies (Lander et al., 1997). Here, we investigate 
the source of the microheterogeneity of the CSPGs and perineuronal nets detected by the Cat-3xx antibodies.

\section{MATERIALS AND METHODS}

Purification of CSPGs. Adult rat cortex and spinal cord were dissected and homogenized in buffer A [4 M guanidine-HCl (Sigma, St. Louis, MO), $50 \mathrm{~mm}$ sodium acetate, $10 \mathrm{~mm}$ EDTA, and $1 \%$ 3-[(cholamidopropyl)-dimethylammonio]-1-propansulfonate, $\mathrm{pH}$ 6.0] with a Dounce homogenizer. After homogenization, samples were stirred on a magnetic stir plate for $8 \mathrm{hr}$ at $4^{\circ} \mathrm{C}$. After stirring, samples were centrifuged at $150,000 \times g$ for $1 \mathrm{hr}$. Lipid floating on top of the soluble fraction was removed by suction. The soluble fraction was removed to a fresh tube and precipitated with $10 \%$ solid trichloroacetic acid (Sigma), and the pellet was discarded. The soluble material was transferred to a fresh tube and precipitated with three volumes of $95 \%$ ethanol with $1.3 \%$ acetic acid. Pellets were resuspended in buffer A and dialyzed into buffer B (4 $\mathrm{m}$ urea, $50 \mathrm{~mm}$ acetate, and $10 \mathrm{~mm}$ EDTA, $\mathrm{pH}$ 6.0) with multiple buffer exchanges. Proteoglycan fractions were then bound to a DEAE-Sepharose column (Amersham Biosciences, Arlington Heights, IL) and eluted in buffer C (buffer B with $1 \mathrm{M} \mathrm{NaCl}$ ) with a continuous gradient. Cat-3xx-reactive fractions were concentrated and dialyzed exhaustively against water for $72 \mathrm{hr}$ with multiple daily buffer exchanges. Samples were aliquoted, dried in a Speed-Vac, and frozen at $-80^{\circ} \mathrm{C}$ until needed.

For sequencing, protein was purified by $\mathrm{CsCl}$ gradients and immunoprecipitated with Cat-315 from cat brains as described previously (Lander et al., 1997). Precipitated protein was purified by SDS-PAGE. Bands were cut from the gel and subjected to tryptic digest. Peptides were isolated by HPLC and sequenced by the Howard Hughes Medical Institute (HHMI)/W. M. Keck Biotechnology Resource Laboratory at Yale University.

Isolation of RNA. Adult rat brain and spinal cord were homogenized, and total RNA was isolated using RNA-stat (Tel-Test, Inc.). Poly $\left(\mathrm{A}^{+}\right.$) RNA selection was performed using oligo-dT columns (Sigma).

Electrophoresis and Western blot analysis. SDS-PAGE on singleconcentration or gradient acrylamide gels was performed using standard techniques. For improved separation of the high molecular weight proteoglycans, a modification of standard SDS-PAGE was used where noted. Modified gels were made of a combination of acrylamide (2.0-4.0\%) and agarose $(0.5 \%)$. All other gel components and methods were identical to those for standard gel electrophoresis. Blots were blocked in 5\% nonfat powdered milk in Tris-buffered saline for $1 \mathrm{hr}$, washed, and incubated with primary antibody diluted in DMEM with $0.5 \%$ Triton X-100 overnight. Blots were washed and incubated with either alkaline phosphataseor HRP-conjugated secondary antibody. Alkaline phosphatase activity was visualized with nitroblue tetrazolium and 5-bromo-4-chloro-3indolyl phosphate. HRP activity was detected using ECL Western blotting following the manufacturer's instructions (Amersham Biosciences).

Deglycosylation. Enzymes for deglycosylation were as follows: chondroitinase ABC, ACII, and B (10 mU/ $\mu \mathrm{l}$; ICN Biochemicals, Costa Mesa, CA), $O$-glycosidase $(0.5 \mathrm{mU} / \mu \mathrm{l}$; Hoffmann-La Roche, Nutley, NJ), sialidase $(10 \mathrm{mU} / \mu \mathrm{l}$; Hoffmann-La Roche), and $N$-glycosidase F (1 U/ $/ \mu$; Hoffmann-La Roche). In sequential digests, samples were first treated with chondroitinase ABC. Dried samples were dissolved in chondroitinase buffer ( $20 \mathrm{~mm}$ Tris and $20 \mathrm{~mm}$ sodium acetate, $\mathrm{pH} 8.0)$ and treated with $2 \mu \mathrm{l}$ of chondroitinase ABC/100 $\mu \mathrm{l}$ of sample. Subsequently, samples were buffer-exchanged into the appropriate buffer for the next digestion in centrifugal filter devices with 30,000 molecular weight cutoff filters (Microcon). For all enzymatic treatments, samples subsequent to chondroitinase $\mathrm{ABC}$ were first denatured by boiling in the appropriate buffer for the enzymes: $50 \mathrm{~mm}$ sodium acetate, $\mathrm{pH}$ 5.0, for sialidase, $O$-glycosidase, or both or $20 \mathrm{~mm}$ sodium phosphate buffer, $\mathrm{pH} 7.2$, for $\mathrm{N}$-glycosidase $\mathrm{F}$ or the combination of $\mathrm{N}$-glycosidase $\mathrm{F}$ with any other enzyme. For all digests, $4 \mu \mathrm{l}$ of enzyme was added per $50 \mu \mathrm{l}$ of sample. Chondroitinase ACII and B were used in conditions identical to those for chondroitinase ABC. All digestions were performed for $12 \mathrm{hr}$ at $37^{\circ} \mathrm{C}$. Reactions were terminated by boiling in SDS gel-loading buffer, and samples were analyzed by Western blotting. On tissue sections, the same enzymes and buffers were used. Tissue sections were first washed in the appropriate buffer and then treated with an enzyme or combination of enzymes for $4 \mathrm{hr}$ at $37^{\circ} \mathrm{C}$. If sections were to be subjected to serial digestion, chondroitinase was always used first and by itself. All other enzymes could be used simultaneously.

For chemical deglycosylations, samples were first purified as described above; however, they were lyophilized for $24 \mathrm{hr}$ to ensure complete dryness into glass tubes with Teflon-lined caps instead of into microfuge tubes. Trifluromethansulfonic acid (TFMS) deglycosylation was performed using the Glyco-Free kit following the manufacture's instructions (Glyko Inc.) with the modifications described below. Samples were deglycosylated for 1,2 , or $4 \mathrm{hr}$ at $-20^{\circ} \mathrm{C}$. After neutralization of the TFMS with pyridine, $50 \mu \mathrm{l}$ of $5 \mathrm{~mm}$ deoxycholate (Sigma) in 50\% TCA was added to samples. Samples were precipitated on ice for $30 \mathrm{~min}$, after which $100 \mu \mathrm{l}$ of methanol was added, and the samples were pelleted by centrifugation. The supernatant was discarded, and the pellet was washed twice with $500 \mu \mathrm{l}$ of cold acetone $\left(-20^{\circ} \mathrm{C}\right)$. Acetone was removed, and the samples were dried for $15 \mathrm{~min}$ in a Speed-Vac to remove any residual acetone. Samples were analyzed by Western blotting.

Purified proteoglycan and tissue sections were deglycosylated by $\beta$ elimination using alkaline sodium borohydride (Sigma). Proteoglycan samples and tissue sections were treated with $0.3 \mathrm{M}$ sodium borohydride in $0.4 \mathrm{M} \mathrm{NaOH}$. Proteoglycan samples were incubated in alkaline borohydride for $4 \mathrm{hr}$ at room temperature and then neutralized by the addition of $1 \mathrm{M} \mathrm{HCl}$, concentrated, and analyzed by Western blots. Tissue sections were incubated for $30 \mathrm{~min}$ at room temperature and then analyzed using standard immunohistochemical techniques (see below). Tissue sections were additionally treated with $10 \mathrm{~mm}$ periodate in $50 \mathrm{~mm}$ sodium acetate, $\mathrm{pH} 4.5$, for $30 \mathrm{~min}$ at room temperature. After oxidation, sections were incubated in $0.3 \mathrm{~m}$ sodium borohydride in $0.4 \mathrm{M} \mathrm{NaOH}$ for $30 \mathrm{~min}$. Samples were washed in phosphate buffer and analyzed by standard immunohistochemical techniques (see below).

$R$ Nase protection assay. Total RNA was isolated from rat cortex and spinal cord as described above. The templates for the probes were produced by reverse-transcribing total RNA from rat adult rat spinal cord using an aggrecan-specific internal primer. Amplification of this product using a $5^{\prime}$ primer containing an SP6 phage promoter sequence and a $3^{\prime}$ aggrecan primer generated a template for transcription of an antisense probe. The in vitro transcription reaction was performed using an SP6/T7 transcription kit (Hoffmann-La Roche) and $\left[{ }^{32} \mathrm{P}\right] \mathrm{UTP}$. A cyclophilin probe was purchased from Ambion and prepared according to the manufacturer's instructions. RNase protection assay (RPA) was performed as described previously (Melton et al., 1984). Each total RNA sample was hybridized at $55^{\circ} \mathrm{C}$ for $20 \mathrm{hr}$ with $4 \times 10^{5} \mathrm{cpm}$ of probe in a final volume of $30 \mu \mathrm{l}$ of hybridization buffer (40 mM PIPES, pH 6.4, 400 $\mathrm{mm} \mathrm{NaCl}, 1 \mathrm{~mm}$ EDTA, and 80\% deionized formamide). Each sample was then treated with $0.27 \mu \mathrm{g}$ of RNase A (Hoffmann-La Roche) and 300 $\mathrm{U}$ of RNase T1 (United States Biochemicals, Cleveland, $\mathrm{OH}$ ) in digestion buffer (in mM: 10 Tris, pH 7.5, 5 EDTA, and $300 \mathrm{NaCl}$ ) for $15 \mathrm{~min}$ at $30^{\circ} \mathrm{C}$. Samples were then treated with $100 \mu \mathrm{g}$ of Proteinase K (United States Biochemicals) and $20 \mu \mathrm{l}$ of $10 \%$ SDS for $15 \mathrm{~min}$ at $37^{\circ} \mathrm{C}$. Samples were then phenol/chloroform-extracted and precipitated with salt and ethanol. Pellets were resuspended in $7 \mu \mathrm{l}$ of gel-loading buffer (Ambion) and electrophoresed on a $6 \%$ polyacrylamide, $8 \mathrm{M}$ urea sequencing gel for $3 \mathrm{hr}$ at $35 \mathrm{~W}$. The gel was then exposed to X-OMAT AR film (Eastman Kodak, Rochester, NY) with an intensifying screen.

In situ hybridization. Frozen sections (12-14 $\mu \mathrm{m}$ thick) were thawmounted onto gelatin-coated slides and postfixed in $0.1 \mathrm{M}$ sodium phosphate-buffered $4 \%$ paraformaldehyde, $\mathrm{pH}$ 7.4. Sections were rinsed in PBS and $2 \times$ SSC and acetylated with $0.5 \%$ acetic anhydride in $0.1 \mathrm{M}$ triethanolamine, $\mathrm{pH}$ 8.0. Sections were then rinsed in $2 \times$ SSC and PBS, dehydrated in ethanols, and delipidated in chloroform. Sections were prehybridized in $2 \times \mathrm{SSC}$ and $50 \%$ formamide at $55^{\circ} \mathrm{C}$ for $1 \mathrm{hr}$ and then hybridized in $0.75 \mathrm{M} \mathrm{NaCl}, 50 \%$ formamide, $1 \times$ Denhardt's solution, $10 \%$ dextran sulfate, $30 \mathrm{~mm}$ DTT, $10 \mathrm{~mm}$ Tris-HCl, pH 7.5, 1 mM EDTA, $100 \mu \mathrm{g} / \mathrm{ml}$ salmon sperm DNA, $0.5 \mathrm{mg} / \mathrm{ml}$ yeast tRNA and $1.5 \times 10^{6} \mathrm{cpm}$ probe per slide at $55^{\circ} \mathrm{C}$ for $12-15 \mathrm{hr}$. $\left[{ }^{33} \mathrm{P}\right] \mathrm{C}$ TP-labeled antisense and sense probes to exons 3 and 12 were synthesized using an SP6/T7 transcription kit (Hoffmann-La Roche) as described above. After hybridization, sections were washed in $2 \times \mathrm{SSC}, 50 \%$ formamide, and $0.1 \%$ $\beta$-mercaptoethanol (BME) at $55^{\circ} \mathrm{C}$ for $1 \mathrm{hr}$ and treated with $20 \mu \mathrm{g} / \mathrm{ml}$ RNase A in $0.5 \mathrm{M} \mathrm{NaCl}$ and $10 \mathrm{~mm}$ Tris- $\mathrm{HCl}, \mathrm{pH} 8.0$, at $37^{\circ} \mathrm{C}$ for $30 \mathrm{~min}$. Sections were washed in $2 \times \mathrm{SSC}, 50 \%$ formamide, and $0.1 \% \mathrm{BME}$ at $60^{\circ} \mathrm{C}$ for $30 \mathrm{~min}$ and $0.1 \times \mathrm{SSC}$ and $0.1 \% \mathrm{BME}$ at $65^{\circ} \mathrm{C}$ for $30 \mathrm{~min}$ and dehydrated. Slides were exposed to film (Hyperfilm; Amersham Biosciences) for 8-11 d.

Immunohistochemistry. Rats were deeply anesthetized and transcardially perfused with PBS followed by ice-cold $4 \%$ phosphate-buffered paraformaldehyde. Forty micrometer frozen sections were cut on a cryostat. Free-floating sections were incubated overnight in primary antibody with $1 \%$ Triton X-100. The following day they were rinsed with phosphate buffer and incubated in HRP-conjugated goat anti-mouse 
secondary antibody (Jackson ImmunoResearch, West Grove, PA) or Alexa fluorescent-conjugated goat anti-mouse secondary antibodies (Molecular Probes, Eugene, OR) diluted in DMEM with 5\% FCS and 1\% Triton X-100 for $2 \mathrm{hr}$. Sections were rinsed in phosphate buffer and reacted with $0.03 \%$ DAB (Sigma) and $0.003 \%$ hydrogen peroxide.

PCR analysis. One microgram of poly $\left(\mathrm{A}^{+}\right)$-selected RNA from the adult rat cortex and spinal cord was reverse-transcribed using oligo-dT. Oligonucleotide primers were made to every exon-exon junction in the aggrecan gene; $3^{\prime}$ probes were made just $3^{\prime}$ to the each exon junction, and $5^{\prime}$ primers were made just $5^{\prime}$ to every exon-exon junction. PCR analysis was performed directly on reverse-transcribed mRNA or using nested primers on preamplified substrates when necessary. The resulting PCRamplified products were analyzed for size by agarose gel electrophoresis. The bands were excised, and the product was cloned into TOPO pCR 2.1 TA-cloning vector following the manufacturer's instructions (Invitrogen, San Diego, CA). Clones were subsequently sequenced by the HHMI/ W. M. Keck Biotechnology Resource Laboratory at Yale University using the fluorescently labeled dideoxynucleotide chain termination method. PCR-amplified products containing confirmed splice variants were cloned into pcDNA3.1/CT-green fluorescent protein-TOPO expression vector following the manufacturer's instructions (Invitrogen).

\section{RESULTS}

\section{Cat-301, Cat-315, and Cat-316 recognize distinct subsets of neurons in the adult rat CNS}

Previous studies have shown that Cat-301, Cat-315, and Cat-316 recognized perineuronal nets on overlapping neuronal subsets in the cat CNS. A parasagittal section of the rat brain labeled with all three antibodies demonstrates that each antibody also identifies distinct subsets of neurons in the rat (Fig. 1A). Cat-301 strongly labeled neurons in the brainstem and spinal cord but few neurons in other areas (Fig. $1 A, B$ ). In marked contrast, Cat-315 and Cat-316 labeled neurons in the forebrain but few in the brainstem or spinal cord. Although the staining patterns of Cat315 and Cat-316 were overlapping, each of these antibodies also labeled distinct sets of neurons. For example, in the somatomotor cortex, Cat-315 labeled many neurons in layers II, III, IV, and VI, whereas Cat-316 labeled few neurons in layers II, III, and VI and many neurons in layers IV and $\mathrm{V}$, and Cat-301 reactivity was essentially absent (Fig. 1C). In the hippocampus, some neurons were labeled by all three antibodies; others were labeled by only two of the antibodies; and some were labeled by only one of the antibodies (Fig. 1D). Higher-magnification images of the hippocampus and cortex labeled with Cat-315, Cat-316, and antiGFAP show that the reactivity for the Cat-3xx antibodies is surrounding neurons (Fig. 1E,F). We found no examples of cells expressing both GFAP and epitopes recognized by any of the Cat-3xx antibodies. Serial immunoprecipitations showed that, as in the cat, the Cat-3xx antibodies identified seven biochemically distinct proteoglycans in the rat (data not shown). All three antibodies recognized CSPGs of nearly identical apparent molecular mass (Fig. 2).

\section{Aggrecan is the CSPG recognized by Cat-301, Cat-315, and Cat-316}

Converging evidence from several different lines of experimentation demonstrated that the Cat-3xx antibodies recognized aggrecan, the large aggregating proteoglycan predominantly characterized in cartilage. First, the Cat-315-reactive protein was purified from adult cat and subjected to amino acid sequence analysis. Two internal peptides generated by tryptic digests were identified as the amino acid sequences KWRISK and HHAFCFR. These peptides are identical to sequences from the $\mathrm{G}_{1}$ and $\mathrm{G}_{2}$ domains of aggrecan, respectively (C. Lander and S. Hockfield, unpublished observations). Neither peptide was identical to any other known protein, providing evidence that Cat-315 recognizes aggrecan in the adult CNS. We next analyzed protein expression in the brain from mice carrying the cartilage matrix deficiency $(\mathrm{cmd})$ mutation. Homozygous $\mathrm{cmd}$ mice express no detectable aggrecan in cartilage and die at birth. Heterozygous cmd mice express a reduced level of aggrecan in cartilage compared with wild-type controls and survive into adulthood (Watanabe et al., 1994, 1997). The brain CSPGs recognized by our antibodies do not reach significant levels until after birth, prohibiting the analysis of homozygous $\mathrm{cmd}$ mice for Cat-3xx expression. Therefore, we assayed antigen expression in adult heterozygous $\mathrm{cmd}$ mice compared with wild-type mice. Quantitative Western blots showed a reduction in immunoreactivity for a known anti-aggrecan antibody, 1-C6, and Cat-301, Cat-315, and Cat-316 in adult heterozygous $\mathrm{cmd}$ mice. Expression of the CSPGs recognized by all four antibodies was reduced nearly $40 \%$ compared with wild-type controls (Fig. 3), providing compelling evidence that all three Cat-3xx antibodies recognize aggrecan.

All three antibodies identify one or two predominant, very high molecular weight bands ( $>500 \mathrm{kDa})$. In addition to the dominant bands, three or four less intensely stained, lower molecular weight bands, between 200 and $450 \mathrm{kDa}$, were also detected (Fig. 2). Like the high molecular weight antigens shown in Figure 3, the less abundant, lower molecular weight species showed a parallel reduction in intensity in $c m d$ mice, suggesting that all bands detected by the Cat-3xx antibodies are products of the aggrecan gene. Recent work has demonstrated substantial proteolytic processing of aggrecan in the CNS (Lemons et al., 2001), and the lower molecular weight species identified here are likely to represent cleavage products of aggrecan. Cat-315 recognizes a high molecular weight proteoglycan in the embryonic rodent brain, which we have established not to be aggrecan through the analysis of homozygous $\mathrm{cmd}$ mice. This "early" Cat-315 reactivity is downregulated over the course of development as aggrecan Cat315 reactivity appears and is entirely absent in the adult (data not shown).

\section{Aggrecan mRNA is expressed late in development and primarily by neurons}

Consistent with our previous demonstration that the Cat-3xxpositive perineuronal net is first detected in the postnatal period (Guimaraes et al., 1990; Hockfield et al., 1990), RNase protection analysis revealed that aggrecan mRNA expression in the cortex and spinal cord is very low at embryonic day 17 (E17) and then rises steadily postnatally to a peak in expression around postnatal day 21 (Fig. 4). The perineuronal net is in direct contact with several cells, a postsynaptic neuron cell body, presynaptic axonal boutons arising from presynaptic neurons, and glial processes that ensheathe the synaptic boutons and extend over the postsynaptic neuron. In situ hybridization was used to determine which of these cells is the likely source of aggrecan in the perineuronal net. In all areas of the brain and spinal cord examined, aggrecan mRNA was detected in specific patterns, nuclei, and cells, which indicated it was expressed predominantly by neurons using two different antisense probes made against sequence from two different aggrecan exons. Expression was restricted to subsets of neurons in most areas of the CNS (Fig. 5). For example, aggrecan mRNA was detected in only some layers and in only a subset of neurons in any given layer of the cerebral cortex and in the CA fields and dentate gyrus of the hippocampus (Fig. $5 A, B$ ). Highermagnification images of the spinal cord demonstrate that aggrecan is highly expressed by motor neurons (Fig. 5D,E). Highmagnification images of the brainstem further demonstrate that 


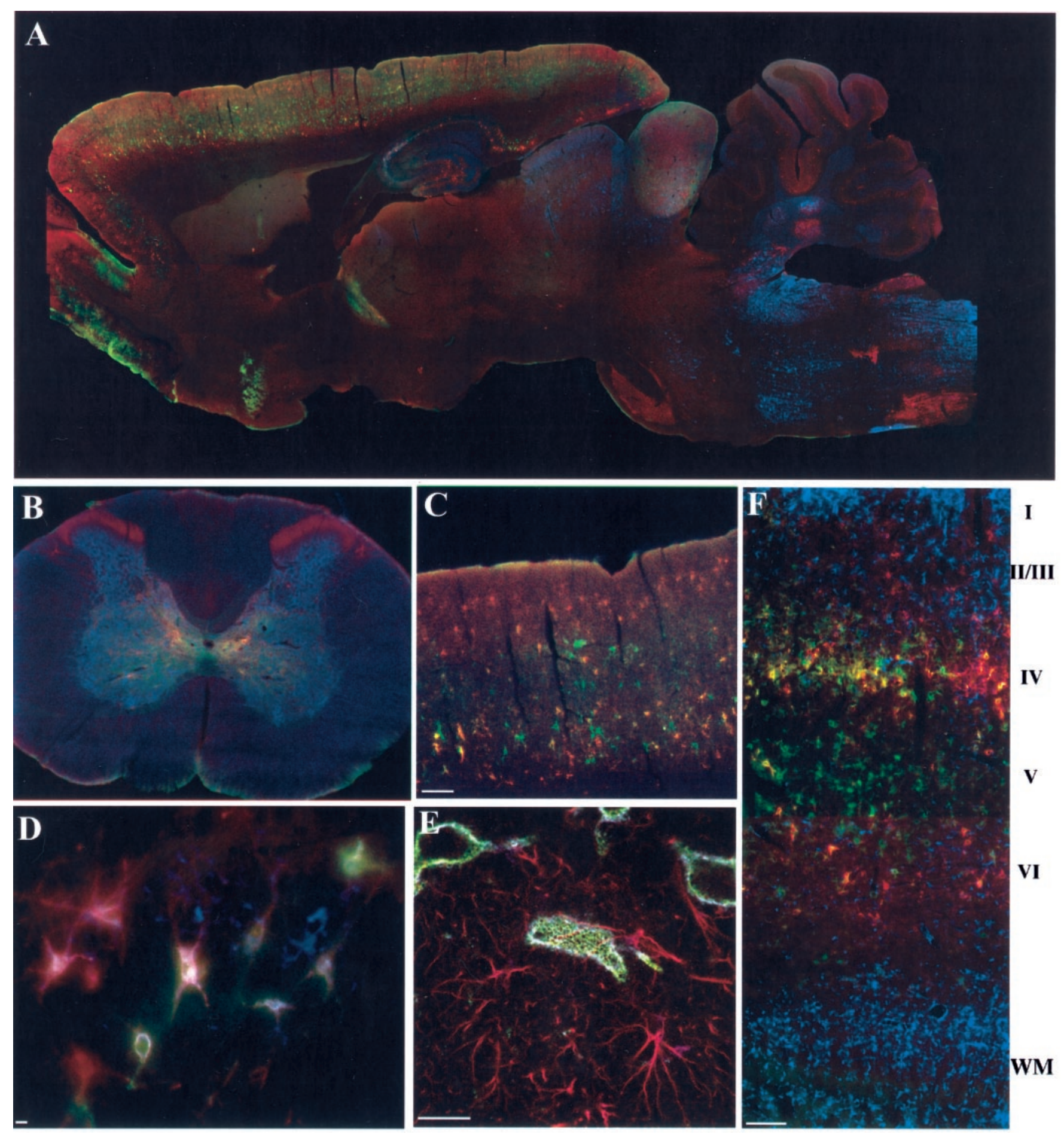

Figure 1. Monoclonal antibodies Cat-301, Cat-315, and Cat-316 recognize different sets of neurons in the rat brain. Cat-301 (blue), Cat-315 (red), and Cat-316 (green) mark the surface of different subsets of neurons in the rat brain, except where otherwise noted. $A$, On a parasagittal section, Cat-301 strongly labels neurons in several brainstem nuclei and in the deep cerebellar nuclei. Cat-315 and Cat-316 show different staining patterns, strongly staining neurons in the reticular nucleus of the thalamus and inferior colliculus and in a laminar and regionally specific pattern in the cortex but staining only a few neurons in the brainstem or spinal cord. $B$, Cat-301 strongly stains neurons in the gray matter of the spinal cord, including motor neurons in the ventral horn and neurons in the dorsal horn. Cat-315-positive neurons are restricted to the midline region and to layer I of the dorsal horn. Cat-316 immunoreactivity mostly overlaps with Cat-301 but is excluded from lateral motor neurons and does not extend into dorsal horn. $C$, Higher magnification of the cortex shows the layer- and cell-specific staining of Cat-315 and Cat-316. Most cortical neurons are stained by only one of these two antibodies. Notice the absence of Cat-301 reactivity. D, Pyramidal neurons at the CAII-III border in the hippocampus are stained with all three antibodies, a combination of two, or only one. E, Higher-magnification confocal images of hippocampal pyramidal neurons and glia labeled with Cat-315 ( green), Cat-316 (blue), and anti-GFAP (red) show that the Cat-3xx epitopes are not expressed on cells labeled with anti-GFAP. $F$, High-magnification image of a somatomotor cortex labeled with Cat-315 (red), Cat-316 ( green), and anti-GFAP (blue). Anti-GFAP reactivity is strong in the molecular layer $(I)$ of the cortex and in the white matter (WM below the cortical layers $(I-I V)$. Cat-315 and Cat-316 reactivity was strong in layers $I I-V I$, with little reactivity in white matter. We found no examples of anti-GFAP-reactive cells that were also reactive with the Cat-3xx antibodies. Scale bars: $C, F, 200 \mu \mathrm{M} ; D, E, 20 \mu \mathrm{M}$. 


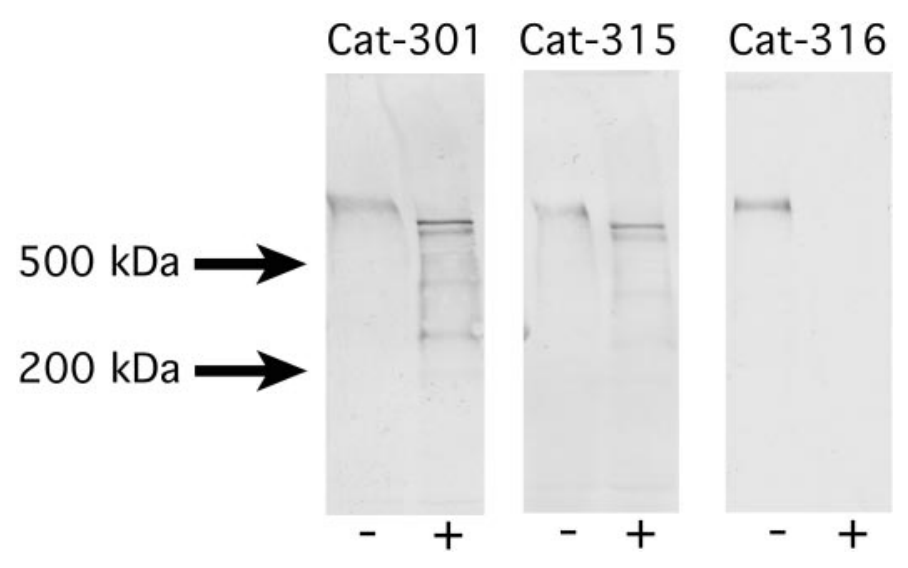

Figure 2. Monoclonal antibodies Cat-301, Cat-315, and Cat-316 recognize CSPGs of similar molecular weights. Rat brain homogenates were incubated with $(+)$ or without $(-)$ chondroitinase. Samples were separated on SDS-PAGE on 3.5-12\% acrylamide gradient gels, electrophoretically transferred, and immunodetected with Cat-301, Cat-315, or Cat-316. The antigens recognized by all antibodies were of the same apparent molecular weight. Treatment of samples with chondroitinase shifted the Cat-301- and Cat-315-reactive bands and abolished Cat-316 staining, consistent with all antigens being CSPGs. Furthermore, these results indicate that Cat-316 recognizes a chondroitin sulfate GAG chain or a mixed protein-GAG epitope.

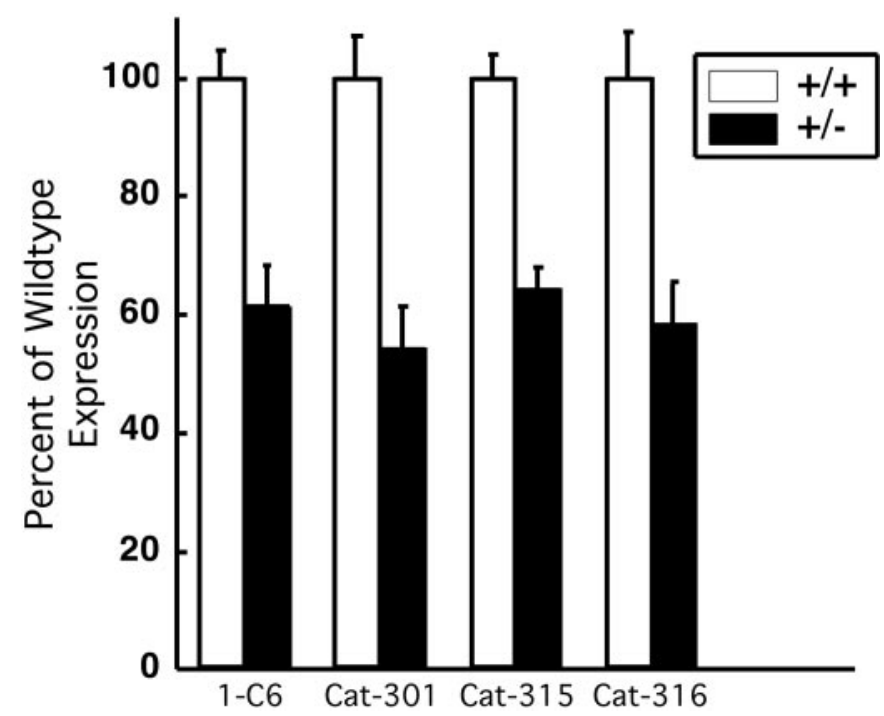

Figure 3. Analysis of brain tissue from $\mathrm{cmd}$ mice indicates that CSPG recognized by the Cat-3xx antibodies is aggrecan. Brain homogenates from adult wild-type $(+/+)$ and heterozygous $(+/-)$ cmd mice were compared by Western blots using antibodies 1-C6 (anti-aggrecan), Cat301, Cat-315, and Cat-316. Reactivity was visualized by ECL and quantified by densitometry. The results are shown as the percentage of reactivity relative to wild-type controls. Reactivity for each of the four antibodies was decreased by $\sim 40 \%$. These results provide strong evidence that the CSPGs recognized by Cat-301, Cat-315, and Cat-316 are aggrecan.

aggrecan mRNA is detected predominately in specific nuclei but not in white matter, consistent with a neuronal origin (Fig. $5 F$ ). Consistent with the results from the RNase protection analysis, we found no detectable aggrecan expression in the brain or spinal cord at E18 despite the presence of a strong signal from aggrecan in cartilage (Fig. $5 H, J$ ). In general, the distribution of neurons that expressed aggrecan mRNA did not resemble the pattern of any one of the Cat-3xx antibodies but roughly matched the distribution of neurons labeled by all three of the antibodies together (Figs. 1, 5). A few regions that showed no reactivity for any of the Cat-3xx antibodies did contain aggrecan-expressing neurons, such as the ventral posteromedial nucleus of the thalamus, substantia nigra, and zona incerta, suggesting the existence of biochemical variants of aggrecan that are not detected with the Cat-3xx antibodies (Figs. 1, 5). Although we cannot rule out the possibility that glia also express aggrecan, we saw no obvious examples of glial aggrecan expression by in situ hybridization.

\section{Cat-3xx aggrecan variants are not derived from alternative splicing}

If all three Cat-3xx antibodies recognize antigens that are products of a single gene, we must account for the biochemical and histological differences detected by the three antibodies. We first asked whether alternative splicing might account for the heterogeneity in aggrecan revealed by the three antibodies. As described above, the spinal cord and cortex show reciprocal staining patterns, with Cat-301 expressed at high levels in spinal cord and at low levels in cortex and Cat-315 and Cat-316 expressed at low levels in spinal cord and at high levels in cortex (Fig. 1). We reasoned that if splice variants accounted for these differences in expression pattern, differential expression of splice variants would be observed in spinal cord and cortex. Reverse transcription (RT)-PCR was performed on cDNA from spinal cord and cortex across each of the 17 exon-exon junctions of aggrecan. This analysis revealed that most aggrecan mRNA in the rat CNS lacks the epidermal growth factor (EGF)-like repeat encoded by exon 13 in both spinal cord and cortex. A very small fraction of aggrecan mRNA $(<5 \%$ ) from rat CNS contained exon 13 (Fig. 6). We did not detect a difference in the expression of the two variants in spinal cord and cortex (Fig. 6). No other splice variants were detected. Furthermore, none of the Cat-3xx antibodies showed reactivity to the $\mathrm{C}$ terminus of aggrecan, with or without the EGF-like domain encoded by exon 13 (data not shown). These results indicate that alternative splicing cannot account for the heterogeneity in aggrecan revealed by Cat-301, Cat-315, and Cat-316.

\section{Cat-316 recognizes chondroitin sulfate GAG chains on aggrecan}

The results from the in situ hybridization analysis of aggrecan expression indicated that each Cat-3xx antibody only detects a subset of aggrecan molecules, but RT-PCR and RNase protection analysis demonstrated that these differences were not a result of alternative gene use. We next investigated whether post-translational mechanisms could account for these biochemical differences. Cat-316 reactivity is abolished by removal of chondroitin sulfate GAGs by digestion with chondroitinase ABC (Lander et al., 1997). In heterozygous $c m d$ mice, Cat-316 reactivity was reduced in parallel with the reduction in immunoreactivity for aggrecan, providing evidence that the GAG recognized by Cat316 modifies few, if any, proteins other than aggrecan. Cat-316, therefore, identifies a particular glycoform of aggrecan. We further investigated the epitope recognized by Cat-316 by enzymatic digestion with chondroitinase ACII and chondroitinase B. Cat316 reactivity was sensitive to treatment with chondroitinase ACII. However, it was completely insensitive to treatment with chondroitinase $\mathrm{B}$ (Fig. $7 A$ ). These results indicate that Cat-316 recognizes a chondroitin sulfate chain and not dermatan sulfate or mixed chains. 


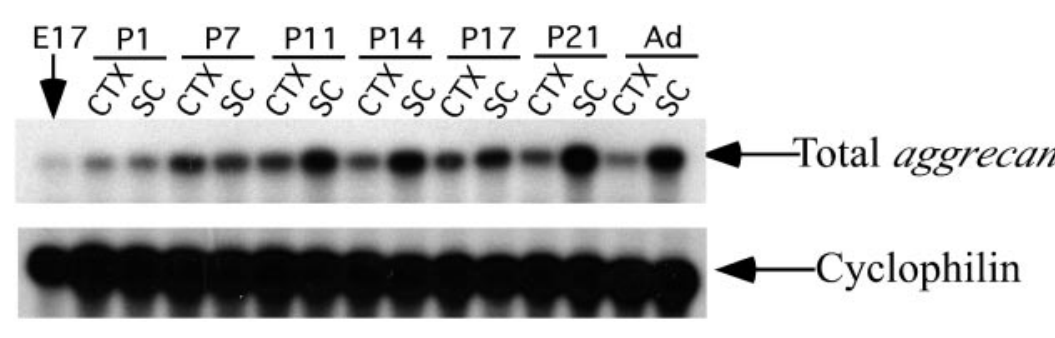

Figure 4. Expression of aggrecan during CNS development. The expression of aggrecan over the course of development was examined in the spinal cord $(S C)$ and cortex $(C T X)$ by RPA. Expression was barely detectable in total brain at E17. Expression increased over the course of development, peaking at postnatal day $21(P 21)$. From $P 21$ through adulthood $(A d)$, aggrecan expression was maintained at a high level in both the spinal cord and the cortex.

\section{Cat-315 recognizes an oligosaccharide epitope on aggrecan, and Cat-301 recognizes a protein epitope on aggrecan that can be masked by oligosaccharides}

Aggrecan from cartilage is modified by all major classes of oligosaccharides, including O-linked GAG chains, other O-linked glycans, and N-linked glycans. We investigated whether these classes of oligosaccharides might generate the differences between Cat315 and Cat-301 reactivity with CNS aggrecan. Chemical and enzymatic deglycosylation demonstrated that Cat-301 and Cat315 also differentially recognize aggrecan glycoforms. TFMS under appropriate conditions hydrolyzes all oligosaccharides from glycoproteins but leaves the polypeptide chain intact. Within $1 \mathrm{hr}$ of TFMS treatment, reactivity for Cat-315 was lost, whereas reactivity for Cat-301 was enhanced (Fig. 7B). These results demonstrate that the epitope for Cat-315, like Cat-316, is an oligosaccharide or requires the presence of a glycan. They further demonstrate that the epitope for Cat-301 resides on the core protein and that this epitope can be masked by oligosaccharides.

\section{The Cat-301 epitope can be unmasked by enzymatic deglycosylation}

Digestion of purified proteoglycan from adult rat brain with chondroitinase ABC, $N$-glycosidase, or sialidase resulted in a decrease in the apparent molecular mass of the Cat-301-reactive protein and a slight increase in the reactivity of the band (Fig. 8). The combination of chondroitinase, sialidase, and $O$-glycosidase led to an additive decrease in the apparent molecular weight of the Cat-301-reactive protein, and the addition of $N$-glycanse to those three enzymes led to an even greater decrease. Combined treatments also led to a more dramatic increase in Cat-301 reactivity. The Cat-301 epitope, therefore, can be blocked by all major classes of oligosaccharides, including GAG chains, sialylated O-linked glycans, and N-linked glycans (Fig. 8). It is likely that the unmasking of the Cat-301 epitope is a specific consequence of the removal of oligosaccharides and not a result of a change in secondary structure, because neither reduction and alkylation nor proteolytic cleavage of aggrecan enhanced (or reduced) the reactivity of Cat-301 for aggrecan or aggrecan peptides (data not shown). The Cat-315-reactive band showed similar shifts in apparent molecular weight as the Cat-301reactive bands, but there was no change in the intensity of staining of Cat-315-positive bands (Fig. 8).

Deglycosylation performed on tissue sections revealed further heterogeneity in neuron-specific expression of aggrecan glycoforms. As described above, each of the three Cat-3xx antibodies identifies a distinct subset of neurons in the adult rat cortex. Few Cat-301-positive neurons but many Cat-315- and Cat-316positive neurons were detected in untreated sections of cortex. Cat-315-positive neurons were found in layers II, III, IV, and VI, and Cat-316-positive neurons were found in layers IV and V (Fig. $1 B)$. After condroitinase digestion, cortical layer $\mathrm{V}$, which contained many Cat-316-positive neurons and few Cat-301- or Cat315-positive neurons in untreated sections, showed a dramatic increase in neoimmunoreactivity for Cat-301 (Fig. 9B). A further increase in Cat-301-neoimmunoreactive cells in layer $\mathrm{V}$ was observed when sialidase and $O$-glycosidase digestion followed chondroitinase digestion; this additional treatment did not give rise to Cat-301-immunoreactive neurons in other layers (Fig. 9C). The addition of $N$-glycosidase had little effect on Cat-301 reactivity (Fig. 9D). In the absence of chondroitinase digestion, $O$-glycosidase and sialidase alone did not produce Cat-301 neoimmunoreactivity in the cortex (Fig. 9E). Cat-315 reactivity was increased by chondroitinase treatment (Fig. 9G), but further enzymatic deglycosylation on tissue sections had no additional effect (Fig. 9F-H), consistent with the results from biochemical deglycosylation experiments (Fig. 8).

\section{Cat-301 reactivity is increased and Cat-315 reactivity is reduced by chemical deglycosylation}

In biochemical assays, TFMS abolished Cat-315 reactivity, demonstrating that Cat-315 recognizes an oligosaccharide. However, the Cat-315 oligosaccharide was resistant to all of the enzymes tested here. To further characterize the oligosaccharide recognized by Cat-315, we used additional deglycosylation methods. $\beta$ elimination catalyzed by a mild base removes O-linked oligosaccharides but leaves $\mathrm{N}$-linked sugars intact. In biochemical assays, $\beta$-elimination removed Cat-315 reactivity, providing evidence that the antibody recognizes an epitope on an O-linked oligosaccharide (Fig. 10). $\beta$-elimination also enhanced Cat-301 immunoreactivity, providing further evidence that the peptide epitope recognized by Cat-301 can be blocked by glycosylation.

Only partial $\beta$-elimination could be achieved on sections; the treatment was too damaging to the tissue to continue it to completion. Partial $\beta$-elimination on tissue sections dramatically reduced Cat-315 reactivity (Fig. $11 E$ ) and enhanced Cat-301 immunoreactivity (Fig. 11B). Cat-301 neoimmunoreactivity in cortex after $\beta$-elimination was again found predominantly in layer $\mathrm{V}$, similar to the results after enzymatic deglycosylation. Cat-315 likely recognizes an epitope on an O-linked sugar (see above); therefore, further characterization of this sugar was attempted using a number of exoglycosidases, including $\alpha$-fucosidase, $\beta$-xylosidase, $\beta$-galactosidase, $\beta$ - $N$-acetyl-hexosaminidase, $\alpha$-galactosidase, and $\alpha$ - $N$-acetyl-glucosaminidase, together with the enzymes described above, in many combinations and sequences. However, no combination decreased Cat-315 reactivity (data not shown), suggesting that Cat-315 recognizes an atypical O-linked sugar.

Cat-315 immunoreactivity could be almost completely eliminated when tissue sections were subjected to oxidation with mild periodate before $\beta$-elimination (Fig. $11 F$ ). This treatment substantially enhanced Cat-301 neoimmunoreactivity, beyond that achieved with any other deglycosylating regimens (Fig. 11C). Cat-301 neoimmunoreactivity appeared not only in layer $\mathrm{V}$ but also on cells in cortical layers II, III, IV, and VI (Fig. 11C). These results, together with the localization of aggrecan RNA by in situ hybridization to neurons in cortical layers II-VI, indicate that as 

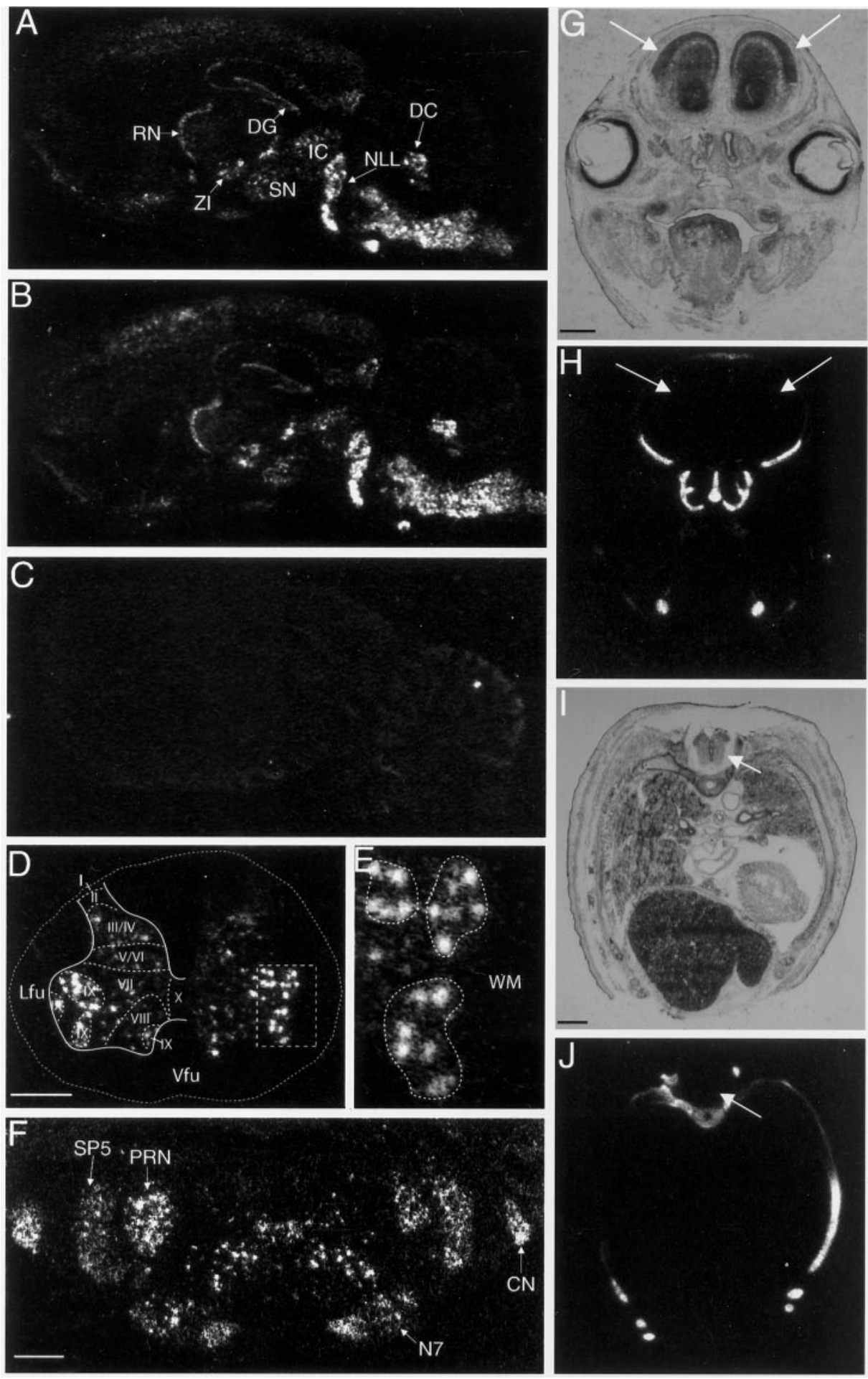

Figure 5. Aggrecan is expressed by neurons in the CNS. In situ hybridization was performed on sections from adult rat brains using a ${ }^{33} \mathrm{P}-\mathrm{labeled}$ antisense probes to exon $12(A, D-F, H, J)$ or exon $3(B)$ of aggrecan. In parasagittal sections $(A, B)$, aggrecan expression was highest in several brainstem nuclei, the deep cerebellar nuclei $(D C)$ and the nuclei of the lateral lemniscus $(N L L)$. High expression was also found in the inferior colliculus $(I C)$, reticular nucleus of the thalamus $(R N)$, zona incerta $(Z I)$, substantia nigra $(S N)$, and dentate gyrus $(D G)$. Aggrecan expression appears to be of neuronal origin; for example, expression is layer-specific in the cortex, CA fields, and dentate gyrus in the hippocampus and in a sharp band in the reticular nucleus. Higher-magnification coronal images of the spinal cord $(D, E)$ and brainstem $(F)$ again demonstrate that aggrecan is expressed by neurons. $D$, In the spinal cord, aggrecan expression is found in the gray matter but is essentially absent in the surrounding white matter. The external border of the spinal cord is marked by a dotted line. The boxed region around lateral motor neurons is shown at higher magnification in E. E, Aggrecan is expressed strongly in spinal motor neurons. Motor neuron pools are circled by dotted lines. Notice the relative absence of aggrecan expression in the surrounding white matter $(W M) . F$, In the brainstem, aggrecan is expressed in particular nuclei, for example, the spinal trigeminal nucleus (SP5), the cochlear nuclei $(C N)$, the parvocellular reticular nucleus $(P R N)$, and the facial nucleus $(N 7)$. We found no obvious examples of aggrecan expression by glia. Transverse sections through the head $(G, H)$ or thorax of an E18 rat embryo were probed for aggrecan $(H, J)$ or stained with cresyl violet $(G, I)$. At E18, there was no detectable aggrecan signal in the brain $(H)$ or spinal cord $(J)$, indicated by arrows, despite strong signal from cartilage aggrecan. $C$, A sense probe (exon 12) shows no signal on a parasagittal section. $L f u$, Lateral funiculus; $V f u$, ventral funiculus. Scale bars, $0.75 \mathrm{~mm}$. 

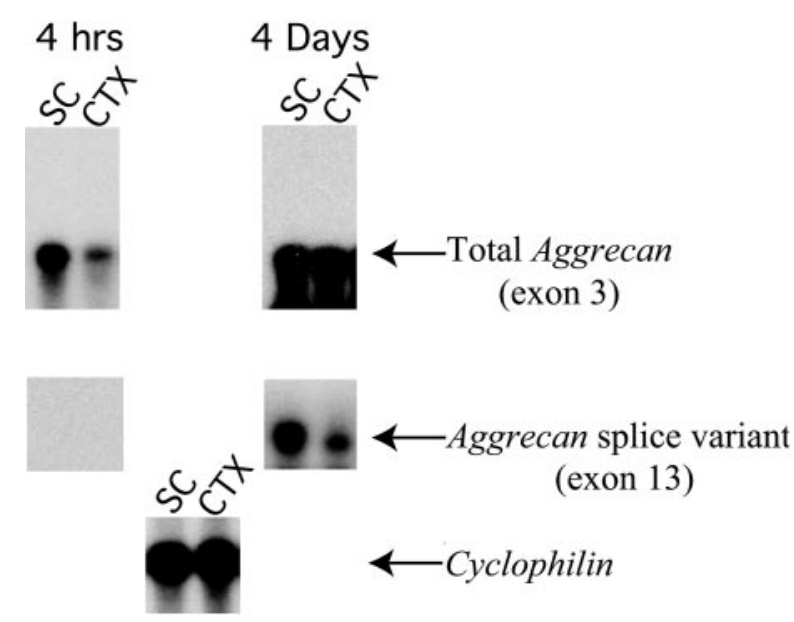

Figure 6. Splice variants do not account for differences in antibody reactivity for aggrecan from different regions of the CNS. The only splice variants of aggrecan detected in the CNS are generated by the alternative usage of exon 13 (the EGF-like domain). The expression of aggrecan with and without exon 13 was analyzed by RPA. Probes were designed to detect both aggrecan isoforms and to detect the isoform that contains exon 13. Expression was evaluated in the adult rat cortex $(C T X)$ and spinal cord $(S C)$. Blots were exposed to film for either $4 \mathrm{hr}$ or $4 \mathrm{~d}$. Both probes show that aggrecan expression is higher in the spinal cord than the cortex. Very little aggrecan transcript containing exon 13 is detected in either spinal cord or cortex; 20 times longer exposures were required to detect the aggrecan isoform that contains exon 13. These studies indicate that aggrecan containing exon 13 is expressed at very low levels in the rat $\mathrm{CNS}$ and that the variation in exon 13 expression does not underlie the staining differences observed among Cat-301, Cat-315, and Cat-316. Cyclophilin was used as a control to indicate equivalent loading of the samples.

deglycosylation moves toward completion, Cat-301 recognizes most variants of aggrecan. Together, the experiments described here show that differential glycosylation of aggrecan gives rise to the distinct patterns of immunoreactivity observed with the three Cat-3xx antibodies.

\section{DISCUSSION}

The present study demonstrates that aggrecan glycoforms are major constituents of perineuronal nets in the adult rat brain. Aggrecan is a member of the lectican family of proteoglycans, a family that includes versican, neurocan, and brain-enriched hyaluronan binding/brevican. Previous studies have shown that lecticans, including aggrecan, are components of perineuronal nets (Yamaguchi, 2000). Aggrecan expression has been demonstrated in the CNS by a number of investigators (Asher et al., 1995; Li et al., 1996; Schwartz et al., 1996; Milev et al., 1998; Lemons et al., 2001) and as a component of the perineuronal net in rats (Asher et al., 1995). Here, we have used in situ hybridization to demonstrate that neurons are the predominant cellular source of aggrecan in the adult CNS, as has been described in chick (Hennig et al., 1992; Schwartz et al., 1996). Aggrecan is expressed in many areas of the CNS, including in the cerebral cortex, the deep cerebellar nuclei, brainstem nuclei, colliculi, spinal cord, dentate gyrus, and CA1, CA2, and CA3 fields of the hippocampus. In each area, the distribution of mRNA expression follows the distribution of neurons with perineuronal nets marked by Cat-301, Cat315 , Cat-316, or a combination. Furthermore, by immunostaining we show that the epitopes recognized by the Cat-3xx antibodies are expressed at least predominantly by neurons, as has been
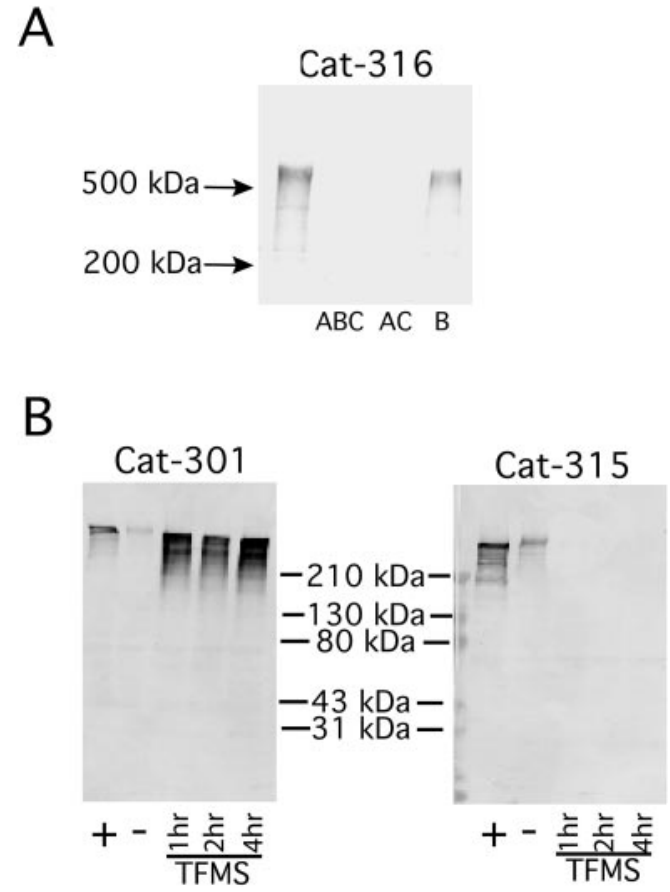

Figure 7. Differences in glycosylation account for the differential reactivity of the Cat-3xx antibodies for aggrecan. $A$, Proteoglycan extracts from rat brains were incubated with chondroitinase $\mathrm{ABC}$, chondroitinase ACII, and chondroitinase B. Samples were separated on SDS-PAGE on $3.5-12 \%$ gradient gels, electrophoretically transferred, and immunodetected with Cat-316. Cat-316 reactivity disappeared after treatment with chondroitinase $\mathrm{ABC}$ and $\mathrm{AC}$ but was unaffected by chondroitinase $\mathrm{B}$. These results indicate that the sugar detected by Cat-316 is a chondroitin sulfate and not a dermatan sulfate or mixed chain. $B$, Samples were deglycosylated by treatment with TFMS for 1,2 , or $4 \mathrm{hr}$, and reactivity to Cat-301 and Cat-315 was analyzed after separation on a $3-20 \%$ polyacrylamide gradient gel. TFMS treatment was compared with chondroitinase ABC-treated (+) and untreated (-) samples. Cat-315 reactivity was increased by chondroitinase treatment but abolished after only $1 \mathrm{hr}$ of TFMS treatment. Cat-301 reactivity was also increased by treatment with chondroitinase but was even more dramatically increased after $1 \mathrm{hr}$ of TFMS treatment. Together these results indicate that the Cat-315 and Cat-316 epitopes are located on carbohydrates and the Cat-301 epitope is located on core protein. Furthermore, the Cat-301 epitope can be blocked by carbohydrate addition.

demonstrated previously. We find no overlapping expression of GFAP and the Cat-3xx antibodies. There has been a longstanding debate over the cellular origin of the components of perineuronal nets (Celio and Blumcke, 1994; Celio et al., 1998). Here, we demonstrate production of aggrecan by neurons, indicating that neurons themselves contribute at least some of the constituents of their perineuronal nets and, furthermore, that neurons can directly regulate the composition of the ECM that surrounds them. Finally, we have demonstrated that the cellspecific composition of perineuronal nets is mediated, at least in part, by variation in aggrecan glycosylation. The differential glycosylation of aggrecan adds substantial heterogeneity to the perineuronal net and represents a robust mechanism for the generation of neuronal diversity.

Using in situ hybridization, RNase protection analysis, and antibody staining, we have demonstrated that aggrecan is not expressed in the CNS until late in embryonic development, peaks in expression at $\sim 1$ month of age, and is maintained at relatively high levels into adulthood. Our data are mostly consistent with 


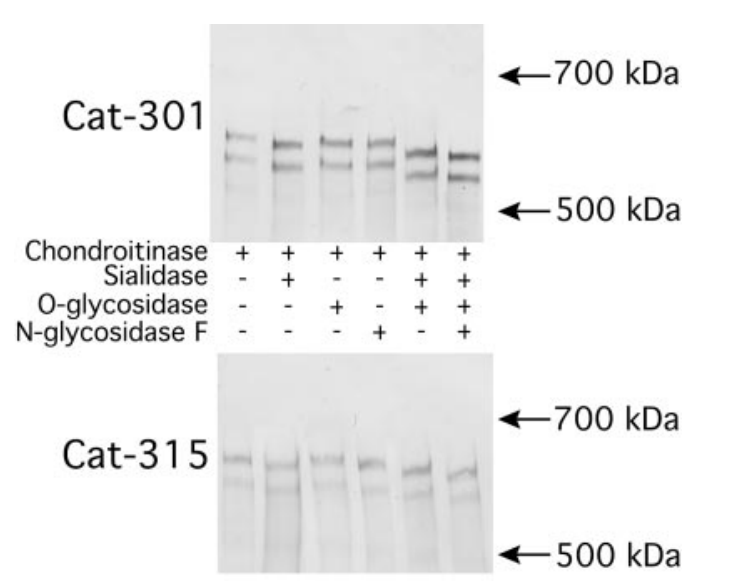

Figure 8. Effects of enzymatic deglycosylation on Cat-301 and Cat-315 reactivity with purified proteoglycans. A brain proteoglycan preparation was treated with chondroitinase $\mathrm{ABC}$ before treatment with other enzymes. Samples were separated on a mixed acrylamide $(2.5 \%)$-agarose $(0.5 \%)$ gel and analyzed by Western blot. Sialidase alone, $N$-glycanse alone, and the combination of sialidase and $O$-glycanse decreased the apparent molecular weight of bands immunoreactive with Cat-301 and Cat-315. In addition, the combination of all three enzymes led to an even more dramatic shift in the apparent molecular weight of the bands detected with each antibody. None of the enzyme treatments had any effect on the intensity of staining with Cat-315. However, each enzyme led to a slight increase in the intensity of Cat-301 staining, and sialidase in combination with $O$-glycosidase or $O$-glycosidase and $N$-glycosidase led to a dramatic increase in staining intensity with Cat-301. These results indicate that Cat-301 can be blocked by GAG chains, sialic acid residues, and other O- and N-linked glycans. Despite the fact that Cat-315 recognizes a carbohydrate, it is a carbohydrate that is not sensitive to the four enzymes used here.

previous work that has analyzed the expression of the aggrecan protein over the course of rat development. Previous work has demonstrated an 18-fold increase in aggrecan reactivity from the embryonic to adult brain (Milev et al., 1998). Milev and colleagues (1998) demonstrated a steady increase in aggrecan protein from 1 to 5 months of age, but we have not found a similar increase. These differences are likely caused by differences in our methods and the fact that although the present analysis focused on mRNA, other studies have analyzed protein. Little is known about the stability and turnover of aggrecan protein or the translational mechanism for aggrecan production in the CNS that may contribute to the differences described here. By in situ hybridization and RNase protection, we detected little aggrecan expression early in development. Previous reports have demonstrated expression of aggrecan protein as early as E14 in the spinal cord (Lemons et al., 2001) and brain (Milev et al., 1998). We did not see any aggrecan expression in the CNS by in situ hybridization, or RNase protection analysis before E19 and E17, respectively. Consistent with these results, using the Cat-3xx antibodies, we detected no protein reactivity until $\sim$ E20 (data not shown). Part of these differences may be attributable to differences in methods as described above; however, we saw no aggrecan message from E14 to E16, which would seem to disallow the presence of the protein at these stages. Future studies will be aimed at resolving these discrepancies.

The developmental pattern of aggrecan expression in the rodent CNS is surprisingly different from that in the chick. In the chick, aggrecan is expressed from embryonic day 7 to day 19 in the CNS but is dramatically decreased at late embryonic stages

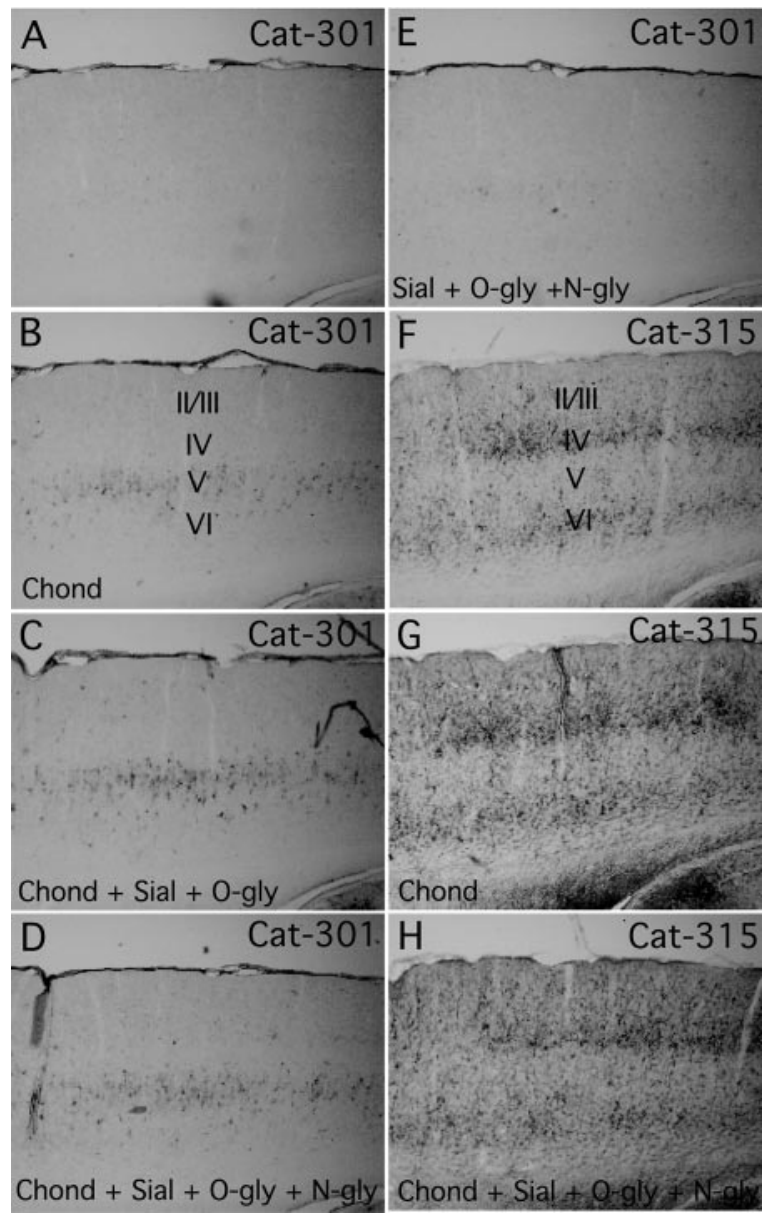

Figure 9. The effects of enzymatic deglycosylation on Cat-301 and Cat315 reactivity in the cortex. Shown are parasagittal sections of somatomotor cortex stained with Cat-301 $(A-E)$ and Cat-315 $(F-H)$ after no treatment $(A, F)$, treatment with chondroitinase (Chond; $B, G)$, treatment with chondroitinase, sialidase (sial), and $O$-glycanse $(O$-gly; $C)$, or treatment with chondroitinase, sialidase, $O$-glycanse, and $N$-glycosidase $\mathrm{F}$ $(N$-gly; $D, H)$ or sialidase, $O$-glycanse, and $N$-glycosidase F $(E)$. Chondroitinase treatment by itself or in combination with any other enzyme reveals Cat-301 neoimmunoreactivity in layer $\mathrm{V}$ of the cortex $(B, C, D)$. The addition of sialidase and $O$-glycosidase increases the neoimmunoreactivity in layer $\mathrm{V}$, but no neoimmunoreactivity is revealed in any other layer, regardless of the combination of enzymes used. Interestingly, sections treated with sialidase, $O$-glycosidase, and $N$-glycosidase F showed very little neoimmunoreactivity in the cortex $(E)$. These results suggest that GAG chain removal is essential for Cat-301 recognition of aggrecan in layer $\mathrm{V}$ of the cortex but is not essential for Cat-301 to recognize aggrecan in other regions, such as the brainstem. Cat-315 reactivity in the cortex was mostly unaffected by any of the enzymes used in this study $(F, G)$.

and is absent after hatching (Hennig et al., 1992; Krueger et al., 1992). In the chick, aggrecan expression coincides with neuronal proliferation and migration and the formation of nuclei (Hennig et al., 1993). In contrast, in the rodent, aggrecan expression is first detected much later in development and more closely follows the establishment and stabilization of synapses. These differences suggest unique roles for aggrecan in the development of the avian and mammalian CNSs.

Previous biochemical studies from this laboratory showed several differences between aggrecan and the Cat-301 CSPG from brain, leading to the suggestion that aggrecan was not the 


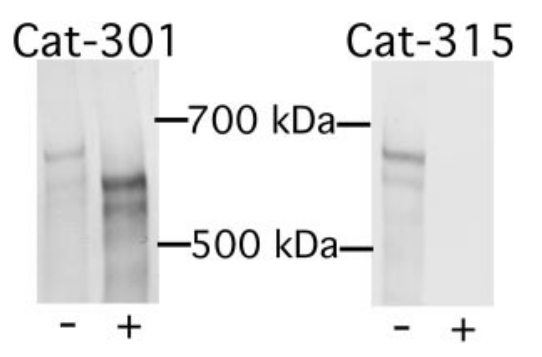

Figure 10. Effects of $\beta$-elimination of purified proteoglycan on Cat-301 and Cat-315 reactivity. Samples were subjected to $\beta$-elimination with alkaline sodium borohydride, a treatment previously shown to specifically remove O-linked glycans. Untreated purified proteoglycans $(-)$ were compared with purified proteoglycans treated with alkaline sodium borohydride $(+)$ and separated on a mixed acrylamide $(3 \%)$-agarose $(0.5 \%)$ gel. Treatment with alkaline borohydride caused a decrease in the apparent molecular weight and an increase in immunoreactivity of the Cat301-reactive band. Conversely, Cat-315 reactivity was abolished by alkaline borohydride treatment. These results confirmed the TFMS results that Cat-301 recognizes aggrecan core protein and Cat-315 recognizes a carbohydrate on aggrecan.

Cat-301 antigen. Aggrecan from cartilage carries keratan sulfate GAG, whereas the Cat-301 antigen from brain does not; in $\mathrm{CsCl}$ density gradients, aggrecan from cartilage distributes in a different fraction from the Cat-301-reactive antigen from the CNS, and antibodies to aggrecan have staining patterns in the CNS that differ from Cat-301 staining patterns (Fryer et al., 1992). The findings reported here, whereas mostly consistent with our previous studies, now reveal that unexpected diversity in glycosylation was responsible for the apparent differences between cartilage aggrecan and the Cat-301 antigen from brain. Other studies have also now shown differences in glycosylation of aggrecan. Aggrecan in chick cartilage carries keratan sulfate GAG chains, whereas aggrecan in chick brain lacks keratan sulfate and contains fewer chondroitin sulfate GAG chains (Li et al., 1996; Schwartz et al., 1996). Aggrecan from chick notochord, but not from chick brain or cartilage, carries the HNK-1 epitope (Domowicz et al., 1995). As discussed further below, aggrecan glycodiversity is likely to be even more complex than revealed in these studies.

The microheterogeneity of glycosylation of a single protein in the CNS shown here is unprecedented in its combined degree and exquisite spatial regulation. We show here that differential aggrecan glycosylation is regulated with regional, laminar, and cell-type specificity. Neural CSPGs with multiple carbohydrate epitopes is not without precedent. For example, phosphacan (also called DSD-1-PG) is heterogeneously glycosylated in the CNS. The presence or absence of keratan sulfate chains discriminates between at least two major glycoforms of phosphacan (Maurel et al., 1994). In addition, phosphacan carries the HNK-1 and Lewis-X epitopes, along with other carbohydrate epitopes (Allendoerfer et al., 1995, 1999; Maeda et al., 1995; Preobrazhensky et al., 1997). It has been suggested that not all phosphacan in the CNS carries Lewis-X structures, because the staining for Lewis-X and phosphacan does not perfectly overlap in the CNS (Allendoerfer et al., 1999). Because these studies used antibodies that recognize epitopes expressed on many different molecules, it has been difficult to definitively discriminate among phosphacan glycovariants. Although phosphacan carries many carbohydrate epitopes, the differential spatial expression of phosphacan glycoforms has

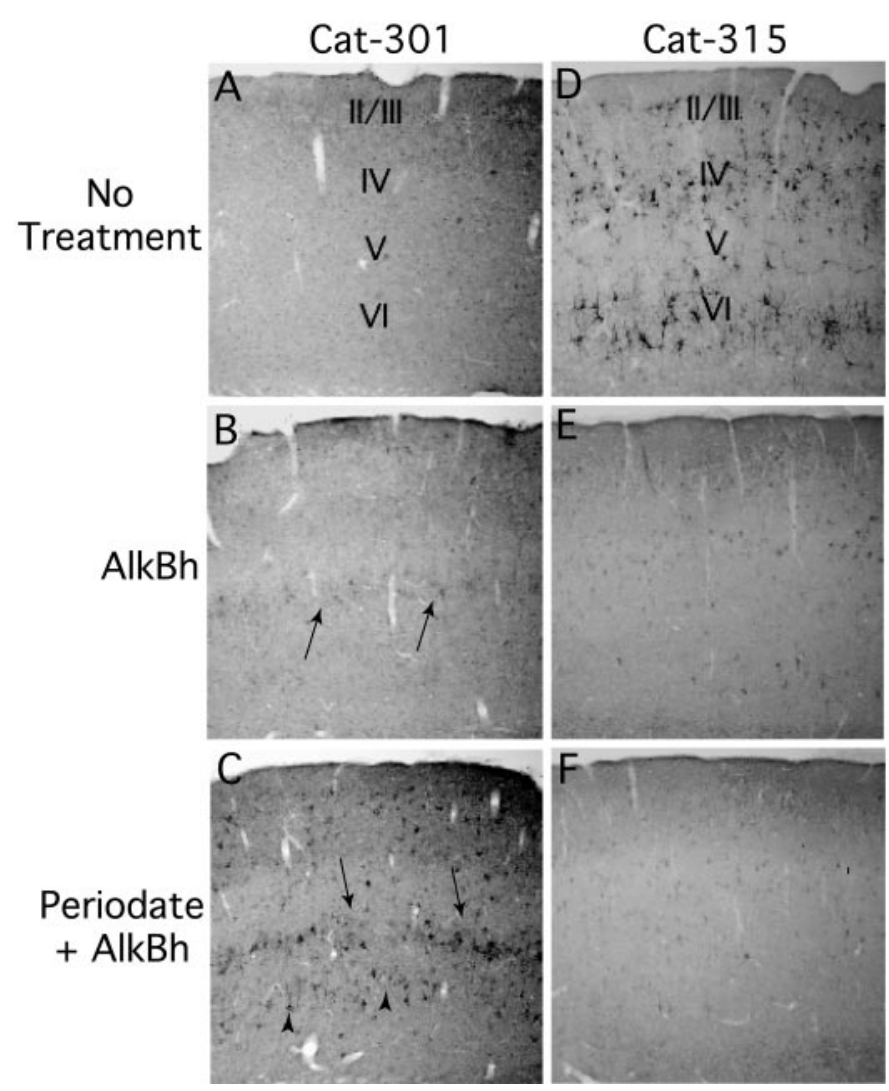

Figure 11. Effects of chemical deglycosylation on Cat-301 and Cat-315 in the cortex. Shown is a section of the somatomotor cortex stained with either Cat-301 $(A-C)$ or Cat-315 $(D-F)$ after no treatment $(A$, $D)$, after treatment with alkaline sodium borohydride $(A l k B h ; B, E)$, and after treatment with periodate followed by alkaline sodium borohydride $(C, F)$. Cortical Cat-301 neoimmunoreactivity was detected in layer $\mathrm{V}$ after treatment with alkaline borohydride, highlighted by arrows $(B)$. Interestingly, as shown in $D$, Cat-315 normally stains neurons in cortical layers IV and VI and not layer V. Treatment of sections with both periodate and alkaline borohydride reveals Cat-301 neoimmunoreactivity in multiple cortical layers, including strong staining in layers V (arrows) and VI (arrowheads) and the detection of staining on scattered cells in layers II-IV $(C)$. Deglycosylation has an opposite effect on Cat-315 reactivity in the cortex. Cat-315 reactivity is decreased by treatment with alkaline borohydride $(E)$ and further reduced by the combined treatment $(F)$.

not been established with the precision we show here for aggrecan. The only other well characterized neural protein that shows regionally specific glycosylation in the CNS is the neural cell adhesion molecule (NCAM). The expression of polysialylated isoforms of NCAM are spatially and temporally regulated (Cremer et al., 2000). However, the microheterogeneity of aggrecan glycoforms, along with the spatial regulation of these glycoforms, far exceeds that described for NCAM and is presently without equal in the CNS.

Over the last decade, descriptions of perineuronal nets have documented a high degree of cell type-specific heterogeneity. Over the same period, data from the human genome project have predicted a surprisingly small number of genes. We show here that differential glycosylation of the protein product of a single gene can give rise to a remarkable degree of biochemical heterogeneity, showing exquisite cell-specific regulation. Even with a limited set of reagents, the present work documents the existence of at least seven different aggrecan glycoforms. In 
situ hybridization reveals neurons that express aggrecan mRNA but that are not recognized by any of the Cat-3xx antibodies, suggesting the existence of additional aggrecan glycoforms.

In every species we have studied thus far, including rat, mouse, cat, monkey, and human, the Cat-315 and Cat-316 antibodies recognize oligosaccharides on aggrecan. In contrast, for many proteins, glycosylation patterns vary significantly between species. Conservation of protein structure across species is a hallmark of functional conservation. If parallel conservation of structure and function holds true for oligosaccharide domains, it might be predicted that Cat-315 and Cat-316 recognize functionally significant domains of aggrecan. Although the functional significance of aggrecan microheterogeneity remains to be explored, aggrecan glycovariants might differentially mediate interactions with other molecules in the extracellular space, including growth factors, adhesion molecules, and other ECM components. The resultant molecular heterogeneity would produce a remarkably large set of neuronal subtypes, defined by the differential composition of the perineuronal nets. How neurons regulate the generation of aggrecan glycoforms is another important question in neuronal cell biology and gene regulation. Families of glycosyltransferases and glycosidases add and remodel carbohydrates on proteins in the endoplasmic reticulum and Golgi complex. The neuron-specific differential glycosylation documented here requires that different neurons express different sets of enzymes, have differential spatial arrangement of enzymes in the secretory pathway, or can vary the availability of substrate sugars. Although we have demonstrated diversity of aggrecan glycosylation, one can predict the existence of additional glycoforms of other CNS glycoproteins. The level of diversity and regulation demonstrated here may have broad implications for understanding the function of glycosylation in the CNS.

\section{REFERENCES}

Allendoerfer KL, Magnani JL, Patterson PH (1995) FORSE-1, an antibody that labels regionally restricted subpopulations of progenitor cells in the embryonic central nervous system, recognizes the Le(x) carbohydrate on a proteoglycan and two glycolipid antigens. Mol Cell Neurosci 6:381-395.

Allendoerfer KL, Durairaj A, Matthews GA, Patterson PH (1999) Morphological domains of Lewis-X/FORSE-1 immunolabeling in the embryonic neural tube are due to developmental regulation of cell surface carbohydrate expression. Dev Biol 211:208-219.

Asher RA, Scheibe RJ, Keiser HD, Bignami A (1995) On the existence of a cartilage-like proteoglycan and link proteins in the central nervous system. Glia 13:294-308.

Bertolotto A, Rocca G, Schiffer D (1990) Chondroitin 4-sulfate proteoglycan forms an extracellular network in human and rat central nervous system. J Neurol Sci 100:113-123.

Bondareff W (1967) An intercellular substance in rat cerebral cortex: submicroscopic distribution of ruthenium red. Anat Rec 157:527-536.

Bruckner G, Brauer K, Hartig W, Wolff JR, Rickmann MJ, Derouiche A, Delpech B, Girard N, Oertel WH, Reichenbach A (1993) Perineuronal nets provide a polyanionic, glia-associated form of microenvironment around certain neurons in many parts of the rat brain. Glia 8:183-200.

Castejon HV (1970) Histochemical demonstration of sulphated polysaccharides at the surface coat of nerve cells in the mouse central nervous system. Acta Histochem 38:55-64.

Celio MR, Blumcke I (1994) Perineuronal nets-a specialized form of extracellular matrix in the adult nervous system. Brain Res Brain Res Rev 19:128-145.

Celio MR, Spreafico R, De Biasi S, Vitellaro-Zuccarello L (1998) Perineuronal nets: past and present. Trends Neurosci 21:510-515.

Cremer H, Chazal G, Lledo PM, Rougon G, Montaron MF, Mayo W, Le Moal M, Abrous DN (2000) PSA-NCAM: an important regulator of hippocampal plasticity. Int J Dev Neurosci 18:213-220.

Domowicz M, Li H, Hennig A, Henry J, Vertel BM, Schwartz NB (1995)
The biochemically and immunologically distinct CSPG of notochord is a product of the aggrecan gene. Dev Biol 171:655-664.

Fryer HJ, Kelly GM, Molinaro L, Hockfield S (1992) The high molecular weight Cat-301 chondroitin sulfate proteoglycan from brain is related to the large aggregating proteoglycan from cartilage, aggrecan. J Biol Chem 267:9874-9883.

Fujita SC, Tada Y, Murakami F, Hayashi M, Matsumura M (1989) Glycosaminoglycan-related epitopes surrounding different subsets of mammalian central neurons. Neuroscience Res 7:117-130.

Guimaraes A, Zaremba S, Hockfield S (1990) Molecular and morphological changes in the cat lateral geniculate nucleus and visual cortex induced by visual deprivation are revealed by monoclonal antibodies Cat-304 and Cat-301. J Neurosci 10:3014-3024.

Hennig A, Krueger R, Mangoura D, Schwartz NB (1992) Chondroitin sulfate proteoglycan expression during neuronal development. Cell Mol Biol (Noisy-le-Grand) 38:585-593.

Hennig AK, Mangoura D, Schwartz NB (1993) Large chondroitin sulfate proteoglycans of developing chick CNS are expressed in cerebra hemisphere neuronal cultures. Brain Res Dev Brain Res 73:261-272.

Hockfield S, McKay RD (1983) A surface antigen expressed by a subset of neurons in the vertebrate central nervous system. Proc Natl Acad Sci USA 80:5758-5761.

Hockfield S, Tootell RB, Zaremba S (1990) Molecular differences among neurons reveal an organization of human visual cortex. Proc Natl Acad Sci USA 87:3027-3031.

Kalb RG, Hockfield S (1988) Molecular evidence for early activitydependent development of hamster motor neurons. J Neurosci 8:23502360.

Kalb RG, Hockfield S (1990a) Induction of a neuronal proteoglycan by the NMDA receptor in the developing spinal cord. Science 250:294296.

Kalb RG, Hockfield S (1990b) Large diameter primary afferent input is required for expression of the Cat-301 proteolycan on the surface of motor neurons. Neuroscience 34:391-401.

Kalb RG, Hockfield S (1994) Electrical activity in the neuromuscular unit can influence the molecular development of motor neurons. Dev Biol 162:539-548.

Krueger Jr RC, Hennig AK, Schwartz NB (1992) Two immunologically and developmentally distinct chondroitin sulfate proteoglycans in embryonic chick brain. J Biol Chem 267:12149-12161.

Lander C, Kind P, Maleski M, Hockfield S (1997) A family of activitydependent neuronal cell-surface chondroitin sulfate proteoglycans in cat visual cortex. J Neurosci 17:1928-1939.

Lander C, Zhang H, Hockfield S (1998) Neurons produce a neuronal cell surface-associated chondroitin sulfate proteoglycan. J Neurosci 18:174-183.

Lemons ML, Sandy JD, Anderson DK, Howland DR (2001) Intact aggrecan and fragments generated by both aggrecanse and metalloproteinase-like activities are present in the developing and adult rat spinal cord and their relative abundance is altered by injury. J Neurosci 21:4772-4781.

Li H, Domowicz M, Hennig A, Schwartz NB (1996) S103L reactive chondroitin sulfate proteoglycan (aggrecan) mRNA expressed in developing chick brain and cartilage is encoded by a single gene. Brain Res Mol Brain Res 36:309-321.

Maeda N, Hamanaka H, Oohira A, Noda M (1995) Purification, characterization and developmental expression of a brain-specific chondroitin sulfate proteoglycan, 6B4 proteoglycan/phosphacan. Neuroscience 67:23-35.

Maurel P, Rauch U, Flad M, Margolis RK, Margolis RU (1994) Phosphacan, a chondroitin sulfate proteoglycan of brain that interacts with neurons and neural cell-adhesion molecules, is an extracellular variant of a receptor-type protein tyrosine phosphatase. Proc Natl Acad Sci USA 91:2512-2516.

Melton DA, Krieg PA, Rebagliati MR, Maniatis T, Zinn K, Green MR (1984) Efficient in vitro synthesis of biologically active RNA and RNA hybridization probes from plasmids containing a bacteriophage SP6 promoter. Nucleic Acids Res 12:7035-7056.

Milev P, Maurel P, Chiba A, Mevissen M, Popp S, Yamaguchi Y, Margolis RK, Margolis RU (1998) Differential regulation of expression of hyaluronan-binding proteoglycans in developing brain: aggrecan, versican, neurocan, and brevican. Biochem Biophys Res Commun 247:207-212.

Nakagawa F, Schulte BA, Spicer SS (1986) Selective cytochemical demonstration of glycoconjugate-containing terminal $\mathrm{N}$-acetylgalactosamine on some brain neurons. J Comp Neurol 243:280-290.

Preobrazhensky AA, Oohira A, Maier G, Voronina AS, Vovk TS, Barabanov VM (1997) Identification of monoclonal antibody At5 as a new member of HNK-1 antibody family: the reactivity with myelinassociated glycoprotein and with two brain-specific proteoglycans, phosphacan and neurocan. Neurochem Res 22:133-140.

Rambourg A, Neutra M, Leblond CP (1966) Presence of a "cell coat" rich in carbohydrate at the surface of cells in the rat. Anat Rec 154:41-71. 
Schwartz NB, Domowicz M, Krueger Jr RC, Li H, Mangoura D (1996) Brain aggrecan. Perspect Dev Neurobiol 3:291-306.

Schweizer M, Streit WJ, Muller CM (1993) Postnatal development and localization of an $\mathrm{N}$-acetylgalactosamine containing glycoconjugate associated with nonpyramidal neurons in cat visual cortex. J Comp Neurol 329:313-327.

Sur M, Frost DO, Hockfield S (1988) Expression of a surface-associated antigen on Y-cells in the cat lateral geniculate nucleus is regulated by visual experience. J Neurosci 8:874-882.

Watanabe K, Oohira A, Katoh-Semba R, Totsuka T, Yoshida K (1989) Sulfated proteoglycans synthesized by Neuro 2a neuroblastoma cells: comparison between cells with and without ganglioside-induced neurites. Neurochem Res 14:707-716.

Watanabe H, Kimata K, Line S, Strong D, Gao LY, Kozak CA, Yamada
Y (1994) Mouse cartilage matrix deficiency (cmd) caused by a 7 bp deletion in the aggrecan gene. Nat Genet 7:154-157.

Watanabe H, Nakata K, Kimata K, Nakanishi I, Yamada Y (1997) Dwarfism and age-associated spinal degeneration of heterozygote cmd mice defective in aggrecan. Proc Natl Acad Sci USA 94:69436947.

Wintergerst ES, Faissner A, Celio MR (1996) The proteoglycan DSD1-PG occurs in perineuronal nets around parvalbumin-immunoreactive interneurons of the rat cerebral cortex. Int J Dev Neurosci 14:249-255. Yamaguchi Y (2000) Lecticans: organizers of the brain extracellular matrix. Cell Mol Life Sci 57:276-289.

Zaremba S, Guimaraes A, Kalb RG, Hockfield S (1989) Characterization of an activity-dependent, neuronal surface proteoglycan identified with monoclonal antibody Cat-301. Neuron 2:1207-1219. 